Bundesgesundheitsbl - Gesundheitsforsch Gesundheitsschutz 2007 · 50:1424-1431 DOI 10.1007/s00103-007-0370-z Online publiziert: 7. November 2007 ๑) Springer Medizin Verlag 2007

A. Moß $\aleph^{1}$ M. Wabitsch ${ }^{1} \cdot$ K. Kromeyer-Hauschild ${ }^{2} \cdot$ T. Reinehr ${ }^{3} \cdot$ B.-M. Kurth ${ }^{4}$

${ }^{1}$ Universitätsklinik für Kinder- und Jugendmedizin, Ulm, BRD . ${ }^{2}$ Universität Jena, BRD .

${ }^{3}$ Universität Witten/Herdecke, BRD $\cdot{ }^{4}$ Robert-Koch-Institut, Berlin, BRD

\title{
Prävalenz von Übergewicht und Adipositas bei deutschen Einschulkindern
}

von $25 \mathrm{~kg} / \mathrm{m}^{2}[1,2]$ und sind damit übergewichtig. Auch im Kindes- und Jugendalter hat die Zahl an Übergewichtigen und Adipösen zugenommen. Dies führt auch zu einem Anstieg Adipositas-assoziierter Komplikationen (körperliche sowie psychische) und zu erheblichen Folgekosten [3]. Die Daten aus dem Kinder- und Ju- gendgesundheitssurvey (KiGGS) des Robert Koch-Instituts (http://www.kiggs.de) geben einen Überblick über die aktuelle Situation in Deutschland. Demnach sind hier $15 \%$ der Kinder und Jugendlichen übergewichtig, was einer Zahl von ca. 1,9 Mio. jungen Menschen entspricht [4]. Auf Basis der Referenzwerte von Anfang

Tabelle 1

Datenerhebung und Datenerfassung zu Übergewicht und Adipositas in den einzelnen Bundesländern: Informationen über Kollektive, Methoden und Quelle. Keine Angaben liegen für Bremen und Sachsen-Anhalt vor

\begin{tabular}{|c|c|c|c|c|}
\hline Land & (n) & Erfassung & Quelle & Definition \\
\hline Baden-Württemberg & 12.573 & Stichprobe & Landesgesundheitsamt BaWü & Kromeyer-H. \\
\hline Bayern & 131.300 & Totalerfassung & Bayerisches Landesamt für Gesundheit und Lebensmittelsicherheit & Kromeyer-H. \\
\hline Berlin & 21.631 & Totalerfassung & Senatsverwaltung für Arbeit, Soziales und Frauen & Kromeyer-H. \\
\hline Brandenburg & k.A. & k.A. & Landesgesundheitsamt Brandenburg im LASV & Kromeyer-H. \\
\hline Hamburg & k.A. & k.A. & 1. Altonaer Gesundheitsbericht & Kromeyer-H. \\
\hline Hessen & k.A. & k.A. & Bayerisches Landesamt & Kromeyer-H. \\
\hline Mecklenburg-Vorpommern & 7.136 & k.A. & Sozialministerium MV & Kromeyer-H. \\
\hline Niedersachsen & 19.487 & k.A. & $\begin{array}{l}\text { Niedersächsisches Landesgesundheitssamt (Hrsg.): Übergewicht } \\
\text { bei Schulanfängern } 2004\end{array}$ & Kromeyer-H. \\
\hline Nordrhein-Westfalen & 151.803 & Totalerfassung & Landesinstitut für den Öffentlichen Gesundheitsdienst NRW & Kromeyer-H. \\
\hline Rheinland-Pfalz & 30.224 & Teilerfassung & $\begin{array}{l}\text { Ministerium für Arbeit, Soziales, Gesundheit, Familie und Frauen; } \\
\text { Referat Gesundheitsberichterstattung }\end{array}$ & Kromeyer-H. \\
\hline Saarland & 10.039 & k.A. & Ministerium für Justiz, Gesundheit und Soziales & Kromeyer-H. \\
\hline Sachsen & k.A. & k.A. & Sächsisches Staatsministerium für Soziales, jugendärztlicher Dienst & Kromeyer-H. \\
\hline Schleswig-Holstein & 28.567 & k.A. & $\begin{array}{l}\text { Kinder- und Jugendärztlicher Dienst der Gesundheitsämter des } \\
\text { Landes Schleswig-Holstein }\end{array}$ & Kromeyer-H. \\
\hline Thüringen & 18.155 & Totalerfassung & $\begin{array}{l}\text { Thüringer Landesamt für Statistik, Öffentlicher Gesundheitsdienst } \\
\text { Thüringen }\end{array}$ & Kromeyer-H. \\
\hline
\end{tabular}

k.A. keine Angabe 
der 1990er-Jahre bedeutet dies einen Anstieg um $50 \%$. 6,3\% der Kinder und Jugendlichen sind adipös (ca. 800.0oo der 1,9 Mio.). Diese Zahl hat sich auf der Basis der o. g. Referenzwerte verdoppelt. Es liegen Trendanalysen aus Einschulungsuntersuchungen vor, die zeigen, dass die Prävalenz von Übergewicht und Adipositas bei Kindern im Einschulungsalter im langfristigen Vergleich zugenommen hat $[5,6,7]$. Ziel dieses Beitrages ist es, eine Übersicht über aktuelle und frühere Befundhäufigkeiten für Übergewicht und Adipositas bei deutschen Einschulkindern in den einzelnen Bundesländern im Vergleich zu geben, Probleme der Datenlage zu benennen und Lösungsmöglichkeiten aufzuzeigen.

\section{Methoden \\ Datenerhebung und Datenerfassung}

Die im Folgenden dargestellten Werte zu Körperhöhe und Körpergewicht wurden im Rahmen der Schuleingangsuntersuchungen der einzelnen Bundesländer erhoben. Der Body-Mass-Index (BMI) wurde berechnet, und daraus abgeleitet wurden die Angaben zu Übergewicht und Adipositas dokumentiert. Die Datenrecherche nach der Häufigkeit von Übergewicht und Adipositas bei Einschülern erfolgte in erster Linie über das Internet und hierbei über die Seiten der Gesundheitsämter, der statistischen Landesämter oder der zuständigen jugendärztlichen Dienste der jeweiligen Bundesländer. Darüber hinaus wurden, wenn nötig, die zuständigen Ansprechpartner ermittelt und per Telefon oder E-Mail kontaktiert (- Tabelle 1). Zusätzlich wurden relevante Publikationen herangezogen $[1,2,3$, $4,5,6,7,8,9,10,11,12,13,14,15,16,17,18,19$, 20, 21, 22, 23]. Zur Vervollständigung der Übersicht und der Darstellung der Prävalenzen insgesamt wurde für die Bundesländer Hamburg, Saarland und Schleswig-Holstein (hier lagen nur getrennte Zahlen zur Prävalenz von Übergewicht und Adipositas bei Jungen und Mädchen vor) der Mittelwert daraus gebildet und als geschätzter Wert angegeben. Dieses Vorgehen ist bei der Interpretation der Daten zu berücksichtigen. Es wurden bei

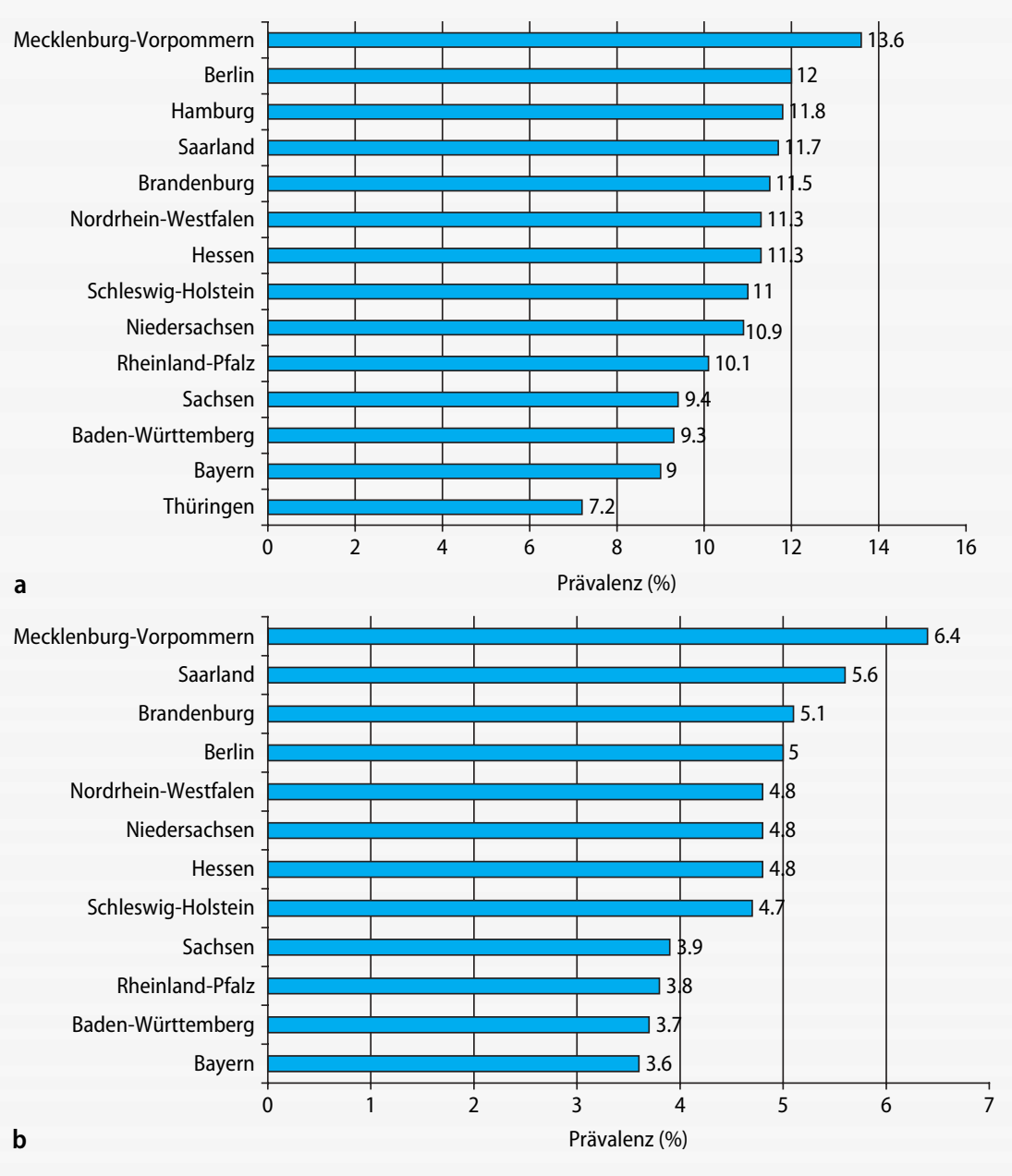

Abb. $1 \Delta$ a Übergewicht bei deutschen Schulanfängern der Einschulungsjahre 2003 (Niedersachsen, Hessen, Mecklenburg-Vorpommern, Brandenburg, Bayern), 2004 (Baden-Württemberg, Berlin, Hamburg, Saarland, Schleswig-Holstein) oder 2005 (NRW, Rheinland-Pfalz, Sachsen, Thüringen). Quelle: Gesundheitsämter der einzelnen Länder, Bayerisches Landesamt für Gesundheit und Lebensmittelsicherheit. $b$ Adipositas bei deutschen Schulanfängern der Einschulungsjahre 2003 (Niedersachsen, Hessen, Mecklenburg-Vorpommern, Brandenburg, Bayern), 2004 (BadenWürttemberg, Berlin, Saarland, Schleswig-Holstein) oder 2005 (NRW, Rheinland-Pfalz, Sachsen). Quelle: Gesundheitsämter der einzelnen Länder, Bayerisches Landesamt für Gesundheit und Lebensmittelsicherheit

den Darstellungen möglichst aktuelle Ergebnisse, meist aus dem Untersuchungsjahr 2004, verwendet.

\section{Definitionen}

Zur Darstellung aktueller Häufigkeiten von Übergewicht und Adipositas wurden die Referenzdaten für den BMI von Kromeyer-Hauschild [24] verwendet. Übergewicht wurde entsprechend den Leitlinien der Arbeitsgemeinschaft Adipositas im Kindes- und Jugendalter (AGA) durch einen $\mathrm{BMI} \geq$ dem 9o. Perzentil definiert. Adipositas wurde durch einen BMI $\geq$ dem 97. Perzentil [25] definiert.

\section{Ergebnisse}

Die Angaben zu Übergewicht und Adipositas sind lückenhaft und sehr uneinheitlich dokumentiert. Darüber hinaus ist der Zugang zu den Daten der Schuleingangsuntersuchungen teilweise schwierig, da die Ergebnisse nicht veröffentlicht sind. In manchen Bundesländern wird routinemäßig eine jährliche Totalerfassung von Größe und Gewicht aller Schulanfänger im Rahmen der Schuleingangsuntersuchung durchgeführt, in anderen erfolgen nur anteilige Erhebungen zu Größe und Gewicht oder sporadische Untersuchungen in unregelmäßigen Abständen. Informationen 


\section{In der Diskussion}

zur sozialen Lage, dem Bildungsstand der Eltern und dem Migrationshintergrund liegen nur vereinzelt vor.

\section{Prävalenz von Übergewicht und Adipositas in den einzelnen Bundesländern}

Die vorliegenden Prävalenzzahlen zu Übergewicht und Adipositas bei Einschulkindern verschiedener Regionen Deutschlands schwanken teilweise erheblich. - Abbildung 1a zeigt die Unterschiede in den aktuellen Prävalenzen von Übergewicht zwischen den Bundesländern: Die Befundhäufigkeit von Übergewicht liegt zwischen $7,2 \%$ in Thüringen und 13,6\% in Mecklenburg. - Abbildung $\mathbf{1 b}$ zeigt die Verteilung der Adipositashäufigkeit in den einzelnen Bundesländern. Auch hier sind regionale Unterschiede festzustellen, wobei sich Prävalenzen finden, die zwischen 3,6\% (in Bayern) und 6,1\% (in Mecklenburg) liegen.

Die zusammenfassende Darstellung der Häufigkeiten für Übergewicht (Adipositas eingeschlossen) bei den Schulanfängern in den einzelnen Bundesländer (• Abb. 2) lässt ein Nord-Süd-Gefälle erkennen. Ein Ost-West-Unterschied ist nicht beobachtbar.

Im Geschlechtervergleich lassen sich relevante Unterschiede nur bei einzelnen Bundesländern feststellen. So ist die Anzahl übergewichtiger und adipöser Jungen z. B. in Mecklenburg-Vorpommern wesentlich höher als die der Mädchen. Im Gegensatz dazu gibt es im Saarland und in Niedersachsen mehr übergewichtige Mädchen als Jungen. In den übrigen Bundesländern sind zwischen Mädchen und Jungen keine Unterschiede in der Befundhäufigkeit zu erkennen (• Abb. 3a und $\mathbf{b})$.

\section{Prävalenz von Übergewicht und Adipositas nach Sozialstatus und Migrationshintergrund}

Für einige Bundesländer (Bayern, Berlin, Brandenburg, Schleswig-Holstein) liegen Befundhäufigkeiten zu Übergewicht und Adipositas bei Einschülern differenziert nach sozialer Schicht und Migrantenstatus vor. Hierbei zeigt sich, dass bei Kindern nicht-deutscher Herkunft der Anteil

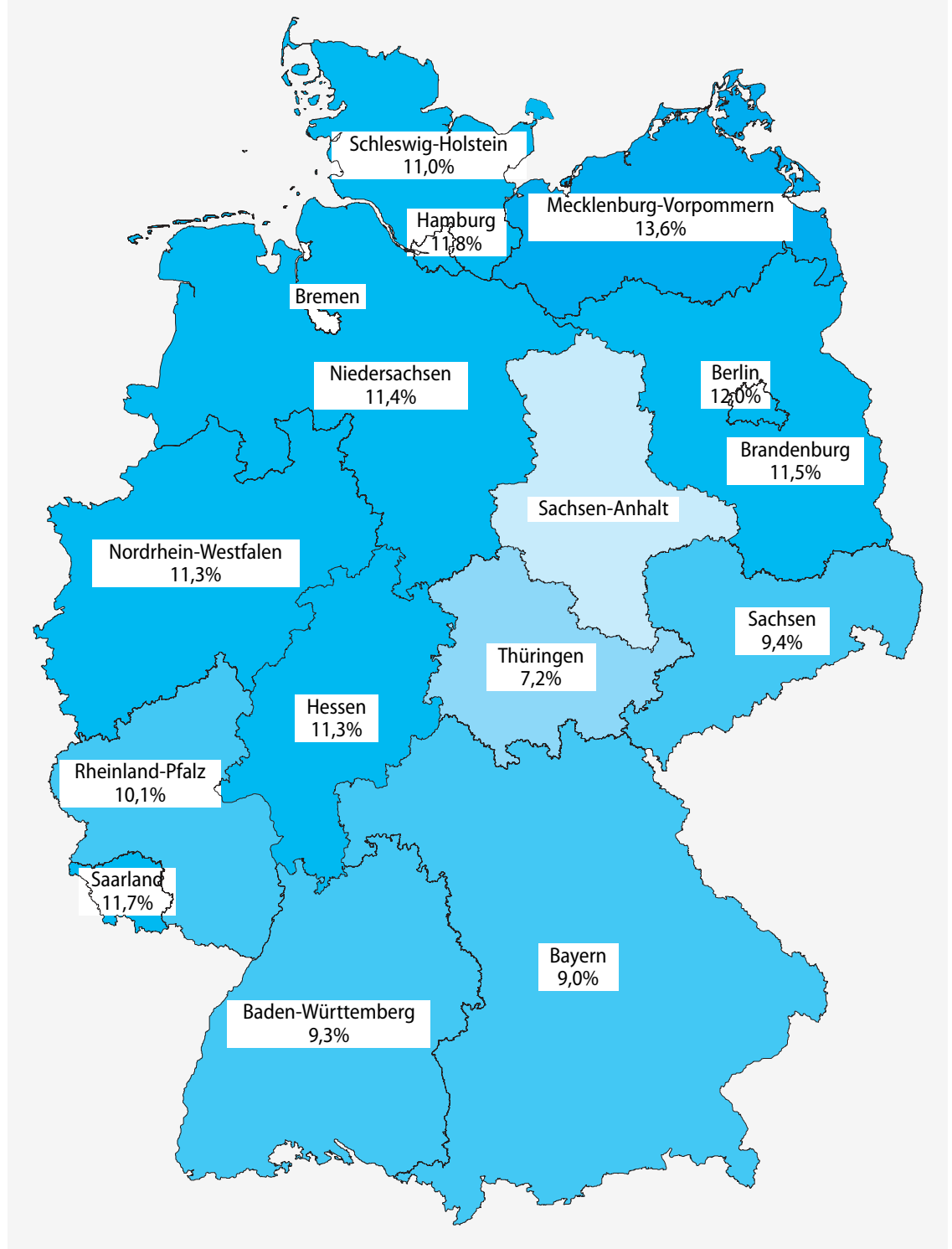

Abb. $2 \Delta$ Überblick über die Prävalenz von Übergewicht bei deutschen Schulanfängern der Einschulungsjahre 2003 (Niedersachsen, Hessen, Mecklenburg-Vorpommern, Brandenburg, Bayern), 2004 (Baden-Württemberg, Berlin, Hamburg, Saarland, Schleswig-Holstein) oder 2005 (NRW, Rheinland-Pfalz, Sachsen, Thüringen). Quelle: Gesundheitsämter der einzelnen Länder, Bayerisches Landesamt für Gesundheit und Lebensmittelsicherheit

Übergewichtiger im Vergleich fast doppelt so hoch liegt, wobei Kinder aus Familien türkischer Herkunft die höchste Prävalenz von Übergewicht aufweisen [7, 9, 10, 11, 12, 13, 14, 15]. Kinder aus der oberen sozialen Schicht sind $\mathrm{zu}$ einem geringeren Prozentsatz übergewichtig als solche aus der unteren $[5,9,15]$. Dies ist bei deutschen Kindern besonders deutlich zu sehen. Bei Kindern türkischer Herkunft unterschieden sich die Anteile Übergewichtiger in den 3 Schichten kaum [11].

\section{Verlaufsdokumentation}

Die Darstellung von Verlaufszahlen zur Prävalenz von Übergewicht und Adipositas bei Einschülern über die letzten Jahre ist für die meisten Bundesländer nicht möglich, da Zahlen aus früheren Untersuchungen entweder nicht vorliegen oder nicht aufbereitet sind. Die Verwendung unterschiedlicher Referenzsysteme erschwert die Beobachtung zeitlicher Trends zusätzlich. Eine solche Auswertung kann nur für wenige Bundesländer präsentiert werden. Im Folgenden wird der Verlauf 
der Befundhäufigkeiten über einen längeren Zeitraum für 2 Bundesländer dargestellt.

- Abbildung 4a zeigt den zeitlichen Trend der Prävalenz von Übergewicht und Adipositas für Nordrhein-Westfalen (NRW) von 1996-2005. In diesem Zeitraum stieg die Häufigkeit von Übergewicht um 1,8\% von 9,5 auf $11,3 \%$, was einen jährlichen Anstieg von 0,10 \% darstellt. 1996 waren 3,9\% der Einschüler in Nordrhein-Westfalen adipös, während 2005 bereits 4,8\% der Schulanfänger an Adipositas leiden. Dies entspricht einem mittleren jährlichen Anstieg von 0,10 \%

In Niedersachsen konnte ein noch deutlicherer Anstieg in Bezug auf die Häufigkeit von Übergewicht und Adipositas zwischen 1993 und 2003 beobachtet werden (• Abb. 4b). Während im Jahre 1993 $8,4 \%$ der Jungen und 9,7\% der Mädchen übergewichtig waren, waren es 2003 bereits $10,4 \%$ bzw. $11,5 \%$. Insgesamt bedeutet dies beim Übergewicht einen Anstieg um $2 \%$ für Jungen und 1,8 \% für Mädchen und damit eine jährliche Zunahme von $0,2 \%$ bzw. 0,18\%. Für die Adipositas sind Anstiege um 1,2\% für Jungen und 1,5\% für Mädchen zu verzeichnen, was einer jährlichen Zunahme von 0,12\% bzw. 0,15\% entspricht. 1993 waren 3,4\% der Jungen und 3,6 \% der Mädchen adipös, während im Jahre 2003 bereits $4,6 \%$ bzw. $5,1 \%$ betroffen waren.

Für Schleswig-Holstein ist zusätzlich eine differenzierte Darstellung der Prävalenzzahlen in den einzelnen Kreisen verfügbar [15]. Es ist festzustellen, dass auch hier bei den Einschülern eine große Variabilität in Bezug auf die Häufigkeit von Übergewicht und Adipositas vorliegt. Es existieren sogenannte "Problemregionen“, wie z. B. Neumünster, mit einem sehr hohen Anteil an übergewichtigen und adipösen Kindern bei einem gleichzeitig sehr hohen Anteil an Migrantenfamilien.

\section{Prävalenz bei älteren Schulkindern}

Für Brandenburg [5] und Thüringen (Thüringer Landesamt für Statistik, Gesundheitsämter) liegen Zahlen zur Prävalenz von Übergewicht bei älteren Schulkindern vor. In beiden Bundesländern steigt die Prävalenz mit zunehmendem Alter der Kinder an, was auch den

Bundesgesundheitsbl - Gesundheitsforsch - Gesundheitsschutz 2007 · 50:1424-1431 DOI 10.1007/s00103-007-0370-z

(c) Springer Medizin Verlag 2007

A. Moß · M. Wabitsch · K. Kromeyer-Hauschild · T. Reinehr · B.-M. Kurth

\section{Prävalenz von Übergewicht und Adipositas bei deutschen Einschulkindern}

\section{Zusammenfassung}

Aus aktuellen Meldungen geht hervor, dass die Übergewichtsproblematik im Kindes- und Jugendalter hoch ist und die Zahl der Betroffenen weiter steigt. Entsprechend den Ergebnissen des Kinderund Jugendgesundheitssurveys (KiGGS) sind $15 \%$ der Kinder in Deutschland übergewichtig und 6,3\% adipös. Der Vergleich mit den Referenzdaten von KromeyerHauschild aus den 1990er-Jahren lässt auf einen deutlichen Anstieg schließen. Eine weitere Informationsquelle zur Darstellung der Prävalenz von Übergewicht und Adipositas im zeitlichen Verlauf sind die regelmäßig durchgeführten Einschulungsuntersuchungen. Eine detaillierte Übersicht über die Prävalenzzahlen zu Übergewicht und Adipositas bei Einschülern der einzelnen Bundesländer war bislang nicht verfügbar. Mittels Internet- bzw. Literaturrecherche sowie Telefon- mit E-Mail-Kontakt wurden die Daten der Schuleingangsuntersuchungen der Bundesländer gesammelt, die Prävalenzangaben zusammengefasst und verglichen. Für Deutschland sind die Prävalenzzahlen zu Übergewicht und Adipositas bei Einschülern sehr uneinheitlich dokumentiert und partiell schwer zugänglich. Die Zahlen aus den verschiedenen Regionen zeigen eine auffallende Heterogenität. So liegt die Befundhäufigkeit für Übergewicht in Thüringen bei 7,2\%, in Bayern bei $9 \%$ und in Mecklenburg-Vorpommern bei 13,6\%. Ähnliche Ergebnisse finden sich für die Adipositasprävalenz mit 3,6\% in Bayern und 6,1\% in Mecklenburg-Vorpommern (berechnet nach Kromeyer-Hauschild et al.). Die zusammenfassende Darstellung der Prävalenzangaben für Schulanfänger lässt ein Nord-Süd-Gefälle erkennen. Im langfristigen Trend ist die Häufigkeit von Übergewicht und Adipositas bei den Schuleingangsuntersuchungen gestiegen. In Brandenburg ist die Adipositasprävalenz seit einiger Zeit rückläufig, auch in einigen anderen Ländern deutet sich neuerdings eine Stagnation der Raten an. Die Schichtzugehörigkeit und der Migrantenstatus sind starke Determinanten für das Auftreten von Übergewicht und Adipositas. Ein Vergleich der Prävalenzen von Übergewicht und Adipositas bei Einschülern mit Ergebnissen aus Schuluntersuchungen im späteren Lebensalter zeigt, dass ein besorgniserregender Anstieg während der Adoleszenz stattfindet. Eine einheitliche und flächendeckende Erfassung und Auswertung der Körperhöhen- und Gewichtsdaten sowie der Werte für den Body-MassIndex (BMI) im Rahmen der Schuleingangsuntersuchung wäre hilfreich, um aktuelle Trends zur Prävalenz von Übergewicht und Adipositas regional differenziert erkennen zu können und um die Wirkung präventiver Maßnahmen im Vorschulalter zu evaluieren.

\section{Schlüsselwörter}

Übergewicht bei Kindern und Jugendlichen · Adipositas · Schuleingangsuntersuchungen - Datenlage 

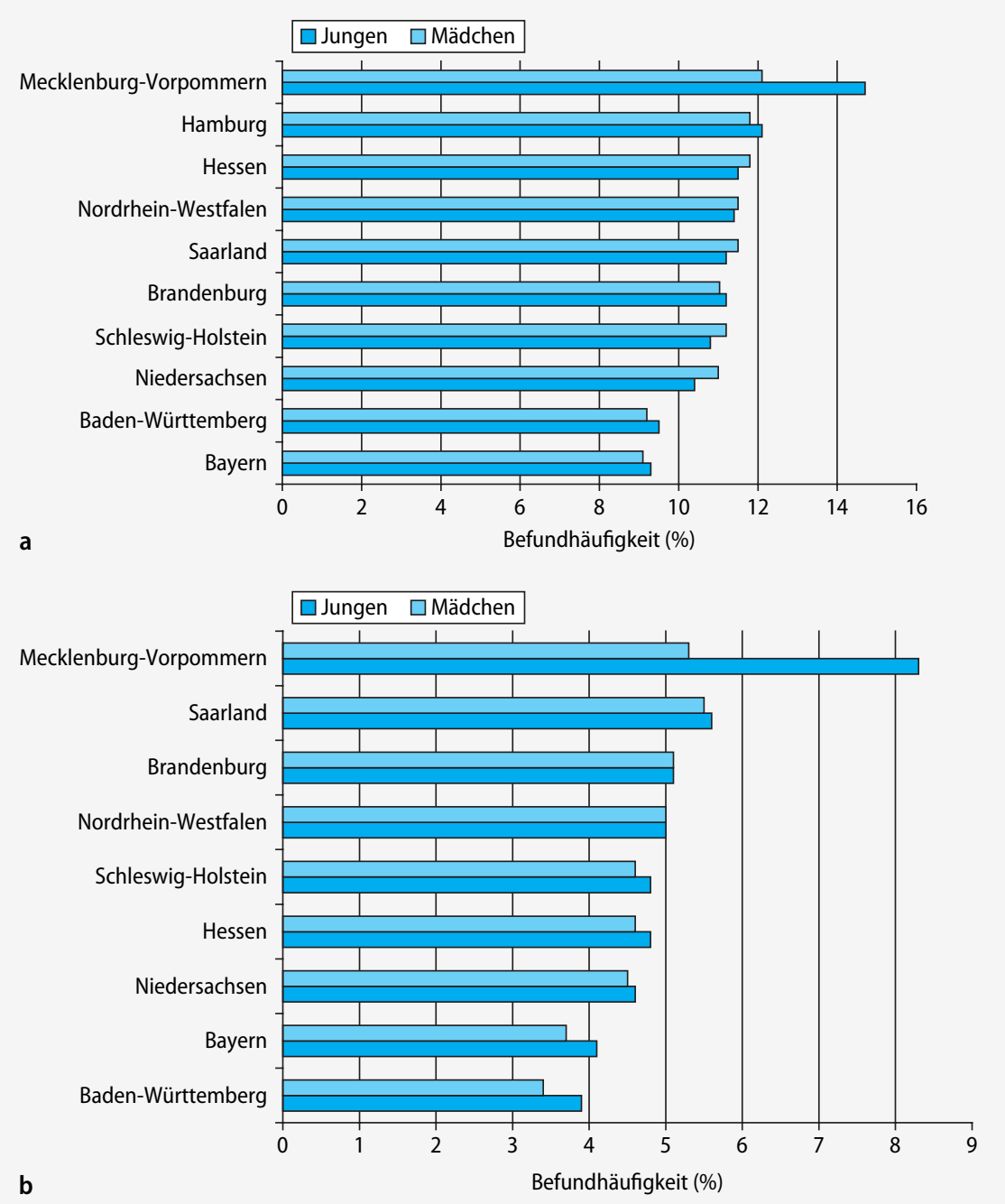

Abb. $3 \Delta$ a Übergewicht bei deutschen Jungen und Mädchen der Einschulungsjahre 2003 (Bayern, Brandenburg, Hessen, Mecklenburg-Vorpommern, Niedersachsen), 2004 (Baden-Württemberg, Hamburg, Saarland, Schleswig-Holstein) oder 2005 (Nordrhein-Westfalen). Quellen: Gesundheitsämter der einzelnen Länder, Bayerisches Landesamt für Gesundheit und Lebensmittelsicherheit. b Adipositas bei deutschen Jungen und Mädchen der Einschulungsjahre 2003 (Bayern, Brandenburg, Hessen, Mecklenburg-Vorpommern, Niedersachsen), 2004 (Baden-Württemberg, Saarland, Schleswig-Holstein) oder 2005 (Nordrhein-Westfalen). Quellen: Gesundheitsämter der einzelnen Länder, Bayerisches Landesamt für Gesundheit und Lebensmittelsicherheit

deutschlandweiten Ergebnissen aus dem KiGGS entspricht [4]. • Abbildung 5 zeigt die Daten für Thüringen. Zusätzlich ist auch ein Anstieg der Prävalenzen von Übergewicht und Adipositas bei 16-Jährigen im Verlauf der letzten 10 Jahre zu verzeichnen [5].

Ähnliche Ergebnisse liegen für das Land Schleswig-Holstein vor, das mithilfe einer eigenfinanzierten KiGGS-Stichprobenaufstockung in der Lage ist, Angaben zur Prävalenz von Übergewicht und Adipositas in den höheren Altersgruppen zu machen. Danach haben 16,9\% der 11- bis 13 -jährigen und $20 \%$ der der 14 - bis 17 - den in Deutschland lebenden Erwachsenen, ist auch bei den Kindern und Jugendlichen bezüglich Übergewicht und Adipositas ein deutliches Nord-Süd-Gefälle erkennbar. Das Beispiel Schleswig-Holstein zeigt zudem, dass die Prävalenzen auch innerhalb eines Bundeslandes variieren können, was sich durch die Unterschiede in der Sozialstruktur oder Bevölkerung der einzelnen Regionen eines Landes erklären lässt. Ähnliche Differenzierungen sind auch innerhalb der Stadtbezirke von Berlin zu finden. Soweit publizierte Informationen verfügbar waren, zeigen diese, dass die soziale Schichtzugehörigkeit und der Migrantenstatus bei Einschülern einen großen Einfluss auf das Auftreten von Übergewicht und Adipositas haben [5, 7, $9,10,11,14,15]$.

Nur von wenigen Bundesländern stehen Zahlen zur Entwicklung der Prävalenz von Übergewicht und Adipositas bei Einschülern im Verlauf der letzten Jahre zur Verfügung; und meist sind diese auch nur schwer zugänglich. Für die wenigen aufbereiteten Zeitreihen ist festzustellen, dass über einen längeren Zeitraum ein stetiger Anstieg von Übergewicht und Adipositas zu verzeichnen war. Dies gilt sowohl für die alten Bundesländer (NRW [16], Niedersachsen [18], Schleswig-Holstein $[12,13,14,15,17])$ als auch für die neuen Bundesländer (Thüringen [6], Brandenburg [8]). Dem gegenüber zeigt sich zumindest in Brandenburg seit dem Jahr 2000 ein Rückgang der Prävalenzzahlen für Adipositas bei Jungen [5]. Bei der Adipositasprävalenz - nicht bei der Übergewichtsprävalenz - in NordrheinWestfalen und auch in Schleswig-Holstein entsteht der Eindruck, dass diese in den letzten 5 Jahren nur langsam angestiegen ist oder sogar stagnierte. Hinweise auf eine längerfristige Stagnation oder sogar auf einen Rückgang konnten wir in anderen Bundesländern nicht finden.

Nach einer Abfrage des Bayerischen Landesamtes für Gesundheit und Lebensmittelsicherheit im Jahr 2007, bei den zuständigen Stellen der Länder, deren endgültige Ergebnisse jedoch noch nicht vorliegen, sind die Adipositasraten (Untersuchungsjahre meist 2005 bzw. 2006) in Bayern, Berlin, Brandenburg, Bremen, Hessen, Mecklenburg-Vorpommern, Nordrhein-Westfalen und im Saarland 

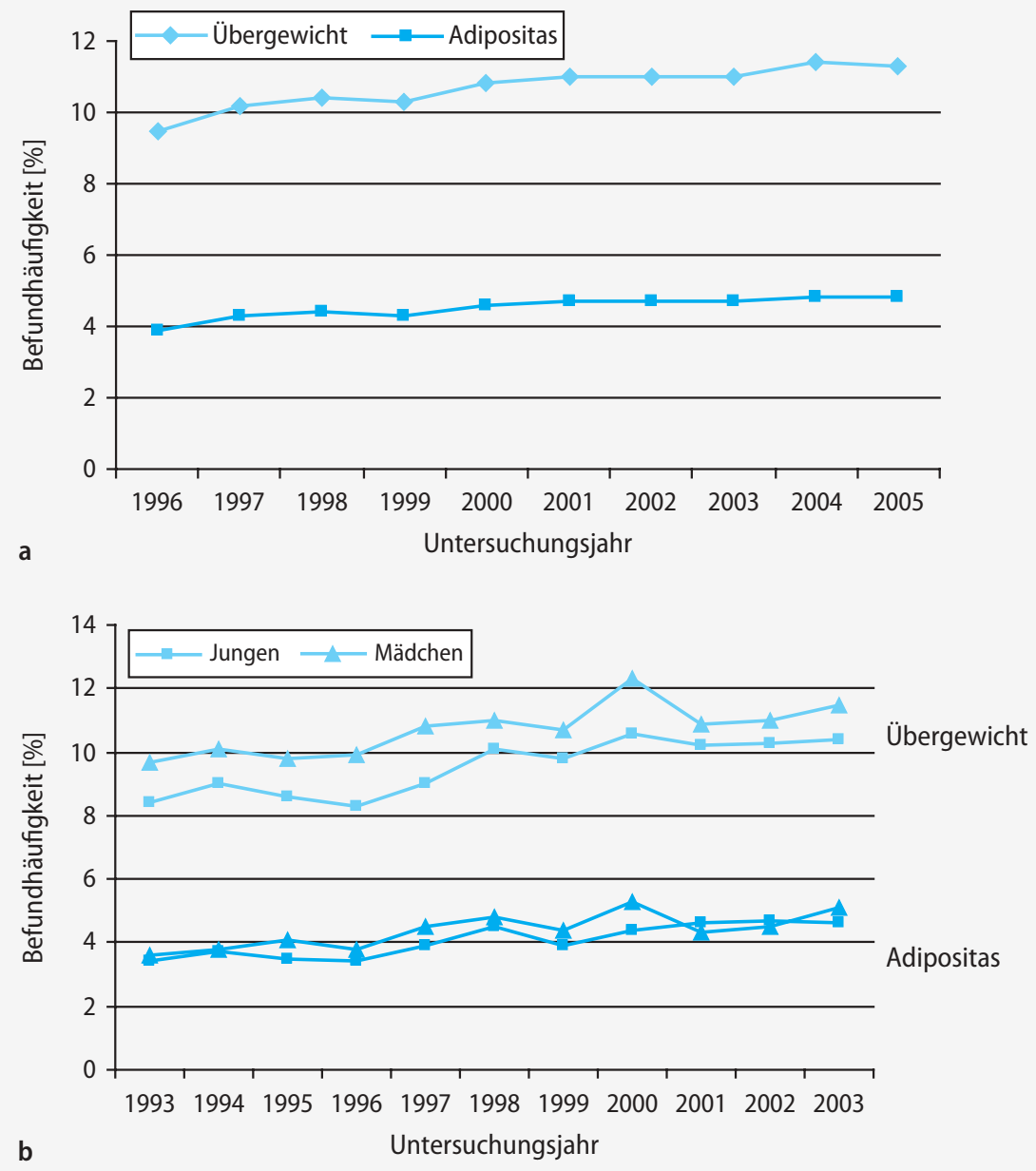

Abb. $4 \Delta$ a Verlauf von Übergewicht und Adipositas bei Schulanfängern in Nordrhein-Westfalen 1996-2005. b Verlauf von Übergewicht und Adipositas bei Schulanfängern in Niedersachsen 1993-2003
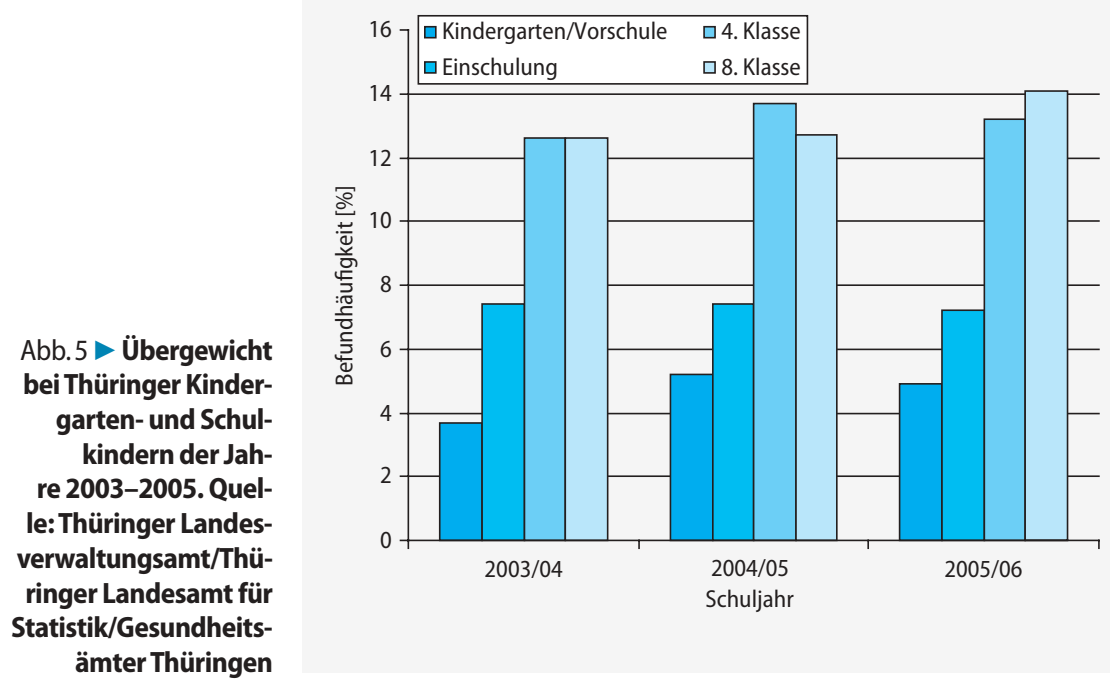

gegenüber der letzten Länderumfrage 2005 (Untersuchungsjahre meist 2003 bzw. 2004) nicht mehr gestiegen. In Niedersachsen sind sie nur bei den Mädchen gestiegen [21]. Jedoch kann aufgrund des kurzen Beobachtungszeitraumes nicht festgestellt werden, ob der Anstieg der Prävalenzzahlen zum Stillstand gekom- men ist. Um eine solche Aussage treffen zu können, wäre die Auswertung von Daten, die über einen längeren Zeitraum erhoben wurden, erforderlich. Dennoch ist dieser Hypothese mit Aufmerksamkeit nachzugehen. Denkbar wäre, dass entgegen aller bislang vorhandenen wissenschaftlichen Evidenz die Kampagnen für mehr Bewegung und gesündere Ernährung im Vorschulalter doch einen Effekt zeigen.

Demgegenüber ist bei den Jugendlichen in allen Bundesländern, von denen Angaben zur Verfügung stehen, in den letzten Jahren ein Anstieg bei den Befundhäufigkeiten zu verzeichnen. Dies gilt auch für Brandenburg. Hier zeigte sich bei den Reihenuntersuchungen der 10. Klassen, dass die Prävalenz von Übergewicht und Adipositas zwischen 1994/95 und 2003/o4 deutlich zunahm [5, 8]. Sollte sich die oben genannte Hypothese einer Stagnation der Prävalenzzahlen für Übergewicht und Adipositas bei Einschülern bestätigen, ist davon auszugehen, dass auf der Basis eines Kohorteneffektes in einigen Jahren kein weiterer diesbezüglicher Anstieg bei älteren Kindern zu verzeichnen sein wird.

Im internationalen Vergleich zeigt sich, dass auch in anderen Ländern ein deutlicher Anstieg in der Prävalenz von Übergewicht und Adipositas bei Kindern und Jugendlichen aufgetreten ist [26, 27, 28]. Dabei deutet sich an, dass die Anstiege in den Befundhäufigkeiten in anderen Ländern höher sind als in Deutschland. In England weisen beispielsweise im Jahr 2004 4-mal mehr Schulkinder ein Übergewicht auf als noch vor 30 Jahren [26]. Auch in den USA, Frankreich und Spanien werden bei Schulkindern steigende Raten für Übergewicht beobachtet [27]. Ein direkter Vergleich mit Verlaufszahlen aus Deutschland ist allerdings nicht möglich, da hier keine repräsentativen Zahlen zur Erstellung einer längeren Verlaufsdokumentation vorliegen.

Ein weiteres interessantes Erhebungsinstrument zur Dokumentation von Veränderungen in den Prävalenzzahlen von Übergewicht und Adipositas bei Schulkindern ist das in Leipzig entwickelte CrescNet-System [29]. Im Untersuchungszeitraum von 1999-2006 wurden Kinder im Alter von 5-7 Jahren standardisiert in teilnehmenden Kinderarztpraxen 


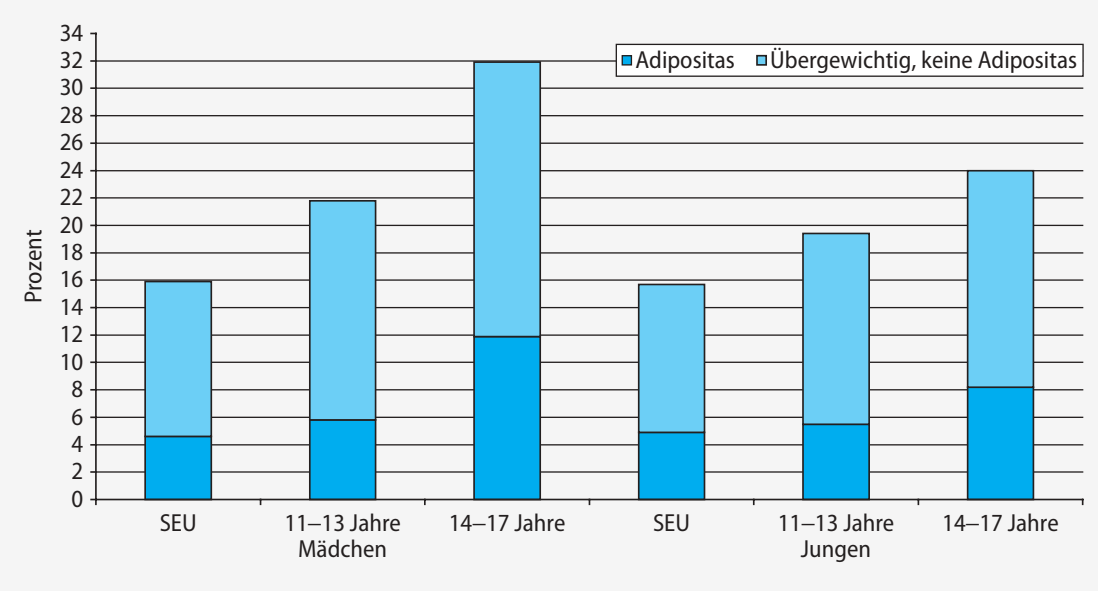

Abb. $6 \Delta$ Prävalenz von Übergewicht und Adipositas bei Kindern und Jugendlichen in SchleswigHolstein nach Altersgruppen und Geschlecht (\%)

untersucht. Im Jahr 1999 waren 11,6 \% der untersuchten Jungen und 10,6 \% der Mädchen übergewichtig. Für die Jungen ist für das Jahr 2006 im Vergleich zum Jahr 1999 ein leichter Rückgang in der Häufigkeit des Übergewichts zu erkennen, während diese bei den Mädchen zunahm (11,1\% Jungen bzw. 12,2 \% Mädchen). Hinsichtlich der Adipositas ist bei den Jungen im Jahresvergleich zwischen 1999 und 2006 kein Unterschied ersichtlich $(4,8 \%$ vs. 4,8\%) während sich bei den Mädchen gestiegene Raten zeigen ( $4,8 \%$ vs. $5,1 \%$ ) (mündliche Mitteilung C. Meigen, CrescNet).

Es muss erwähnt werden, dass die hier vorliegende Darstellung und Diskussion der Prävalenzzahlen von Übergewicht und Adipositas bei Einschulkindern in Deutschland mit dem nötigen $\mathrm{Maß}$ an Vorsicht zu betrachten ist. Die Interpretation der Ergebnisse ist durch das Fehlen einer über alle Bundesländer standardisierten Datenerhebung limitiert. Eine solche Standardisierung der Messmethoden (z. B. Messung mit oder ohne Bekleidung) gibt es nicht. Zudem unterscheiden sich in den verschiedenen Bundesländern die Zeitpunkte, zu denen die Messungen (bezogen auf das Alter der Kinder) durchgeführt werden.

Es muss auch berücksichtigt werden, dass der hier für die Definition der Adipositas bzw. des Übergewichts verwendete BMI kein idealer Indikator für den Gewichtsstatus bzw. die Fettmasse ist. Er reflektiert nicht nur das Körperfett, sondern auch die Masse an Knochen, Muskeln und anderem Gewebe. Entsprechend können Unterschiede in der biologischen Reifung in der Altersphase zwischen 5 und 7 Jahre Untersuchungen zum Auftreten von Übergewicht und Adipositas deutlich beeinflussen [30]. Eine postulierte frühere biologische Reife in den nördlichen Bundesländern würde also theoretisch zu einer Überschätzung der Prävalenzzahlen führen.

\section{Fazit}

Die vorliegenden Daten lassen den Schluss zu, dass die Prävalenz von Übergewicht und Adipositas bei Einschülern in den einzelnen Bundesländern Deutschlands sehr unterschiedlich ist und ein Nord-Süd-Gefälle vorliegt. Angaben zu langfristigen Trends sind nur in wenigen Bundesländern möglich. Hier zeigt sich dann seit Beginn der 1990er-Jahre ein kontinuierlicher Anstieg in der Zahl an übergewichtigen und adipösen Einschülern. In einigen Bundesländern lassen jedoch neueste Untersuchungen die Hypothese zu, dass ein Stillstand in Bezug auf den Prävalenzanstieg erreicht wurde.

Die hier erfolgte Zusammenfassung der aktuell verfügbaren Daten zur Prävalenz von Übergewicht und Adipositas bei Einschülern in Deutschland ist ein erster Schritt zu ihrer regelmäßigen Betrachtung und Diskussion. Zukünftig könnte das Instrument zur Erhebung dieser Zahlen im Rahmen der Einschulungsuntersu- chungen - ggf. gemeinsam mit einer Fortführung des KiGGS - genutzt werden, um weitere mögliche Einflussgrößen auf die Gewichtsentwicklung im Vorschulalter zu identifizieren. Ebenso ließen sich die erhobenen Daten dazu verwenden, die Wirksamkeit von Präventionsmaßnahmen im Vorschulalter zu überprüfen. Die Vereinheitlichung bzw. Abstimmung der Messmethoden und Messzeitpunkte ist eine wichtige Voraussetzung, um die wissenschaftliche Qualität der erhobenen Daten zu verbessern. Entsprechend bemüht sich der Fachausschuss für Kinder- und Jugendmedizin des Bundesverbandes der Ärzte und Zahnärzte des öffentlichen Gesundheitsdienstes bereits seit längerem um eine solche Standardisierung. Die Umsetzung dieser Bemühungen wird aber durch die unterschiedliche gesetzliche Verankerung der Schuleingangsuntersuchungen in den Bundesländern erschwert.

\section{Danksagung}

Die Autoren danken den Mitarbeitern der verschiedenen Behörden für ihre Bemühungen und insbesondere Frau Krämer (Landesgesundheitsamt Baden-Württemberg) und Herrn Rick (Ministerium für Arbeit, Soziales, Gesundheit, Familien und Frauen Rheinland-Pfalz) für die Bereitstellung von Daten sowie Herrn Kuhn (Bayerisches Landesamt für Gesundheit und Lebensmittelsicherheit) für Hinweise auf die vorläufigen Ergebnisse einer laufenden Länderumfrage.

\section{Korrespondierender Autor}

\section{Prof. Dr. M. Wabitsch}

Universitätsklinik für Kinder- und Jugendmedizin

Sektion Pädiatrische Endokrinologie und Diabetologie

Eythstraße 24

89075 Ulm, BRD

E-Mail: martin.wabitsch@uniklinik-ulm.de

\section{Literatur}

1. Benecke A, Vogel H (2003) Übergewicht und Adipositas. Gesundheitsberichterstattung des Bundes Heft 16. Robert Koch-Institut, Berlin

2. Lampert T (2007) Übergewicht und Adipositas in Deutschland. Epidemiol Bull 18:155-156 
3. Wabitsch M, Kunze D, Keller E, et al. (2002) Adipositas bei Kindern und Jugendlichen in Deutschland. MMW Fortschritte der Medizin 120:99-106

4. Kurth BM, Schaffrath Rosario R (2007) Die Verbreitung von Übergewicht und Adipositas bei Kindern und Jugendlichen. Bundesgesundheitsblatt 50:736-743

5. Böhm A, Lüdecke K (2005) Adipositas bei Einschülern und Schülern der 10. Klassen. Trends über 10 Jahre und Zusammenhänge mit der sozialen Lage. Poster 55. Kongress des ÖGD Bamberg

6. Kromeyer-Hauschild K, Zellner K, Jaeger U, Hoyer $\mathrm{H}$ (1999) Prevalence of overweight and obesity among school children in Jena (Germany). Int J Obes 23:1-8

7. Kalies H, Lenz J, von Kries R (2002) Prevalence of overweight and obesity and trends in body mass index in German pre-school children, 1982-1997. Int J Obes 26:1211-1217

8. Böhm A., Friese E, Greil H, Lüdecke K (2002) Körperliche Entwicklung und Übergewicht bei Kindern und Jugendlichen. Analyse von Daten aus ärztlichen Reihenuntersuchungen des öffentlichen Gesundheitsdiensts im Land Brandenburg. Monatsschr Kinderheilkd 150:48-57

9. Delekat D (2007) Zur gesundheitlichen Lage von Kindern in Berlin - Ergebnisse und Handlungsempfehlungen auf Basis der Einschulungsuntersuchungen 2001. Senatsverwaltung für Gesundheit, Soziales und Verbraucherschutz Berlin

10. Bayerisches Landesamt für Gesundheit und Lebensmittelsicherheit (2004) Übergewicht und Adipositas bei Kindern in Bayern. Gesundheitsmonitor Bayern. Eigenverlag, München

11. Gesundheitsberichterstattung Berlin. Senatsverwaltung für Arbeit, Soziales und Frauen. (SenGesSozV) Spezialberichte 2001-1/2003-2/2006-1

12. Thyen U: in Ministerium für Soziales, Gesundheit, Familie, Jugend und Senioren des Landes Schleswig-Holstein (Hrsg) Bericht über die Untersuchungen des Kinder- und Jugendärztlichen Dienstes in Schleswig-Holstein (2001), Erhebung durch den Kinder- und Jugendärztlichen Dienst der Gesundheitsämter des Landes Schleswig-Holstein

13. Thyen U: in Ministerium für Soziales, Gesundheit, Familie, Jugend und Senioren des Landes Schleswig-Holstein (Hrsg) Bericht über die Untersuchungen des Kinder- und Jugendärztlichen Dienstes in Schleswig-Holstein (2002), Erhebung durch den Kinder- und Jugendärztlichen Dienst der Gesundheitsämter des Landes Schleswig-Holstein

14. Thyen U: in Ministerium für Soziales, Gesundheit, Familie, Jugend und Senioren des Landes Schleswig-Holstein (Hrsg) Bericht über die Untersuchungen des Kinder- und Jugendärztlichen Dienstes in Schleswig-Holstein (2003), Erhebung durch den Kinder- und Jugendärztlichen Dienst der Gesundheitsämter des Landes Schleswig-Holstein

15. Thyen U, Brehm S, Meyer C, et al. (2004) Einschulungsuntersuchungen des Kinder- und Jugendärztlichen Dienstes in Schleswig-Holstein

16. Rapp K, Schick KH, Bode H, Weiland SK (2005) Type of kindergarten and other potential determinants of overweight in pre-school children. Public Health Nutr 8:642-649

17. Beiträge zur Gesundheitsberichterstattung des Bundes (2007) Gesundheit von Kindern und Jugendlichen in Schleswig-Holstein. RKI, Berlin

18. Bahrdehle D (2006) Adipositas in NRW. Landesinstitut für den Öffentlichen Gesundheitsdienst NRW. http://www.lögd.de
19. Landesinstitut für den Öffentlichen Gesundheitsdienst NRW (Hrsg) Schulärztliche Untersuchungen in Nordrhein-Westfalen. Jahresberichte 20012005. http://www.lögd.de

20. Bruns-Phillips E, et al. (2004) Adipositas-Bericht 2004. Übergewicht bei Schulanfängern. Eine Auswertung von Schuleingangsuntersuchungen 1993-2003. Niedersächsisches Landesgesundheitsamt Gesundheitsberichterstattung, Hannover

21. Kuhn J (2007) Adipositas: Berichterstattung zwischen Aufklärung und Vernebelung. Gesund in Bayern, Infodienst der Landeszentrale für Gesundheit in Bayern e.V., Mai/Juni 2007: 4-7 http://www. Izg-bayern.de

22. Morlock G, et al. (2006) Ergebnisse der Schuleingangsuntersuchung zum Schuljahr 2004/2005 Statistisch-epidemiologischer Bericht. Bayerisches Landesamt für Gesundheit und Lebensmittelsicherheit, München

23. Duschek KJ, Weinmann J, Böhm K, et al. (2006) Leben in Deutschland - Ergebnisse des Mikrozensus 2005. Statistisches Bundesamt, Wiesbaden

24. Kromeyer-Hauschild K, Kunze D, Wabitsch M, et al. (2001) Perzentile für den Body-Mass-Index für das Kindes- und Jugendalter unter Heranziehung verschiedener deutscher Stichproben. Monatsschr Kinderheilkd 149:807-818

25. Kunze D, Wabitsch M (2006) Leitlinien der Arbeitsgemeinschaft Adipositas im Kindes- und Jugendalter (AGA) der Deutschen Adipositas-Gesellschaft. Verabschiedet auf der Konsensuskonferenz der AGA am 06.10.2006. http://www.a-g-a.de

26. Rigby N. IOTF Press Release May 12 2004. http:// www.iotf.org

27. Lobstein T, Rigby N, Leach R (2005) Obesity in Europe -3 International Obesity TaskForce March 2005. http://www.iotf.org

28. IOTF Childhood Obesity Report May 2004. http:// www.iotf.org

29. Keller E, Gausche R, Meigen C, et al. (2002) Auxological computer based network for early detection of disorders of growth and weight attainment. J Pediatr Endocrinol Metab 149-156. http://www. crescnet.org

30. Wabitsch M (2000) The acquisition of obesity: insights from cellular and genetic research. Proc Nutr Soc 59:325-330

\section{Nadelstichverletzungen kosten 47 Millionen Euro jährlich}

Auch kleinste Stiche können gefährliche Erreger wie das Hepatitis-B-Virus, das Hepatitis-C-Virus oder das HI-Virus übertragen. Aber nicht nur das Gesundheitsrisiko ist sehr hoch. Nadelstichverletzungen kosten viel Geld.

Jede Nadelstichverletzung muss gemeldet werden - schon allein, um den Versicherungsschutz der Mitarbeiter zu garantieren. Nach einer gemeldeten Nadelstichverletzung muss unter Umständen sowohl das Blut des Patienten als auch des Arztes, der sich gestochen hat, auf Krankheitserreger untersucht werden. Besteht ein Infektionsverdacht, so wird der Arzt vorsorglich behandelt. Das alles kostet Geld, vom Arbeitszeitausfall ganz zu schweigen. Durchschnittlich kostet jede gemeldete Nadelstichverletzung $487 €$.

Bei 50.000 gemeldeten Nadelstichverletzungen pro Jahr allein in deutschen Kliniken entstehen dadurch Kosten in Höhe von 24 Millionen Euro. Aber auch wenn die Nadelstichverletzung nicht gemeldet wird, kann es teuer werden. Die meisten Nadelstichverletzungen bleiben ohne Folgen. Hat sich der Mitarbeiter oder die Mitarbeiterin aber doch mit dem Hepatitis-C-Virus infiziert, so kostet die Behandlung in den nächsten 27 Jahren $23.000 €$. Alles zusammen genommen entsteht durch Nadelstichverletzungen jährlich ein Schaden von 47 Millionen Euro.

Gleichzeitig ist wirkungsvoller Schutz vor diesen gefährlichen Verletzungen immer billiger zu haben. Denn heute stehen Arbeitsinstrumente zur Verfügung, die Nadelstichverletzungen fast vollständig ausschließen. Bald sind die sicheren Instrumente kostenneutral.

Eine gute Nachricht auch für Klinikbetreiber, denn seit August 2006 sind in vielen Arbeitsbereichen sichere Instrumente Pflicht. 